\title{
3 Reformulierung der Ausgangsfrage und Forschungsziele
}

Ausgangspunkt der vorliegenden Untersuchung war ein identifizierter Widerspruch von gesundheitspolitischen Konzeptionen und gesellschaftlichen Diskursen zur klinischen Praxis bei schwerstkranken und sterbenden Menschen mit der Frage, ob und wenn ja, wie medizinische Akteure im Krankenhaus diesen Widerspruch wahrnehmen. Bei der Forderung einer Integration der Palliativversorgung in den stationären Bereich zeigt sich im gegenwärtigen Stand der Forschung zudem eine Wissenslücke hinsichtlich der organisatorischen und sozialen Bedingungen sowie der Folgen für das berufliche Ethos und den Behandlungsauftrag im Krankenhaus.

Vor allem zeigt sich in der referierten Literatur eine Forschungslücke über die Versorgungssituation der Mehrzahl der Sterbenden in Deutschland. Denn es sind vorrangig Untersuchungen spezialisierter palliativmedizinischer Behandlungsorte wie Palliativstationen oder Hospize, welche die Behandlung Sterbender thematisieren [5, 119]. Im Vergleich des Auftretens sind jedoch Hospize mit $5 \%$ und Palliativstationen mit 1-2 \% die Versorgungsorte mit den geringsten Sterbefällen, auch wenn sie eindeutig definierte Sterbeorte sind. Denn die meisten Menschen, ca. $50 \%$, sterben auf Normal- und Intensivstationen eines Akutkrankenhauses [2, 33]. Die sogenannte 'Sterbestudie' von George aus dem Jahr 2013 ist die umfassendste und aussagekräftigste Untersuchung der Versorgungsbedingungen für Sterbende in deutschen Akutkrankenhäusern aus Sicht der Behandler [50]. Die Ergebnisse belegen eine Fehlversorgung im Sinne einer Über- oder Unterversorgung Sterbender. Im Anschluss an diese Ergebnisse möchte ich mit einem qualitativen Forschungsansatz die Gründe für die unzureichende Versorgung im gegenwärtig bedeutendsten Versorgungssektor für Sterbende herausarbeiten.

Neben dem Behandlungsort Akutkrankenhaus als identifiziertes Desiderat der Forschung zeigt sich eine weitere Forschungslücke in der Auswahl der tatsächlich Beteiligten an der Behandlung Schwerstkranker und Sterbender in Deutschland. Bisher wurde vorrangig die Perspektive der kleinen Gruppe spezialisierter palliativmedizinisch arbeitender Ärzt*innen untersucht [5, 249, 298, 301, 303, 308]. Ausnahmen bilden die Arbeiten von Wettreck [252], George [50], Kaluza \& Töpferwein [36] und Borasio et al. [255] mit Fokus auf Ärzt`innen außerhalb der spezialisierten Palliativversorgung. Daran anschließend wende ich mich mit der Frage nach der Erfahrung mit der Behandlungssituation schwerstkranker und sterbender Patient*innen an die große und medizindisziplinär heterogene Gruppe der Stationsärzt`innen im Krankenhaus aufgrund ihrer zentralen Rolle in der Krankenbehandlung.

Die Krankenbehandlung im Krankenhaus zeigt sich als komplexer Prozess und zudem als paradigmatisch für die Entwicklung im deutschen Gesundheitssystem [13, 116]. Für die Untersuchung dieses vielschichtigen Themas ist ein exploratives Forschungsdesign gegenstandsangemessen, um die „Situiertheit aller Wissenspro- 
duzent/innen“, die Adele Clarke aufzeigt, anzuerkennen [324, S. 212]. Im Sinne eines qualitativen Forschungsstils expliziere ich noch vor einem Blick in die empirischen Daten meine Präkonzepte als sensibilisierende Konzepte für die Untersuchung [325]. Sie sind als heuristische Konzepte notwendigerweise im Forschungsprozess zu reflektieren. Aus der Rezeption der Rahmenbedingungen und des Forschungsstandes sowie meiner klinischen Erfahrungen im Forschungsfeld Krankenhaus formuliere ich zusammenfassend folgende Präkonzepte:

- Ärztinnen und Ärzte haben im arbeitsteiligen und hierarchischen System Krankenhaus eine Schlüsselposition für die Verbesserung der Behandlung Schwerstkranker und Sterbender inne.

- Eine Behandlung im Krankenhaus hat eine primär als kurativ festgelegte Intention. Wenn Patient*innen im Krankenhaus sterbend sind, zeigt sich eine Verunsicherung über den medizinischen Behandlungsauftrag.

- Behandlungsentscheidungen von Ärzten und Ärztinnen bei palliativen Patient*innen und bei Sterbenden im Krankenhaus basieren auf diffusem rechtlichen, fachlichen und organisatorischen Wissen.

- Im Kontext der gegenwärtigen gesellschaftlich, gesundheitspolitisch, rechtlich und ethisch geführten Debatte über das Lebensende, eines Paradigmenwechsels in der Medizin vom Wohl zum Willen von Patienten und Patientinnen, sowie einer ökonomisierten Krankenbehandlung ist die ärztliche Behandlung Schwerstkranker und Sterbender ethisch-moralisch aufgeladen.

Die Präkonzepte werden im Verlauf des Forschungsprozesses zu gegenstandsbegründeten Hypothesen über das Untersuchungsthema weiterentwickelt. Hier liegt die Stärke qualitativer Forschung, die Erkenntnisse und Theoriebildung über eine Hypothesenprüfung deduktiver Methodologien hinaus anstrebt [326, S. 69ff]. Mit der expliziten Benennung meiner Präkonzepte mache ich auf den dialektischen Prozess von theoriegeleiteter Wahrnehmung und induktiver Datenanalyse aufmerksam [327, S. 38].

Als Forscherin gehe ich nicht ohne Vorwissen in eine Untersuchung, wie es ein naiv-induktives Forschungsverständnis unterstellt. Oder, wie Franz Breuer formuliert: Ein „voraussetzungsloses Entstehen gedanklicher Strukturen bzw. Ideen aus einer Wimmelwelt von (Sinnes-)Daten ist eine erkenntnislogische Unmöglichkeit“ [328, S. 160f]. Meine theoretische Sensibilität, also „die Verfügbarkeit brauchbarer heuristischer Konzepte, die die Identifizierung theoretisch relevanter Phänomene im Datenmaterial ermöglicht“ [329, S. 32], führt zu neuer Erkenntnis. Sie entsteht durch Kenntnisnahme von Literatur, Berufs- und Lebenserfahrungen. Vor diesem Hintergrund stelle ich folgende Fragen an das Datenmaterial:

- Welche Arbeitsbedingungen und Kontextfaktoren beschreiben Ärztinnen und Ärzte für die Behandlung Schwerstkranker und/oder Sterbender im Krankenhaus? 
- Wie erleben und erklären Ärztinnen und Ärzte unterschiedlicher medizinischer Fachdisziplinen die Behandlungskultur bei nicht heilbaren und/oder sterbenden Patient*innen im Krankenhaus?

- Welche Behandlungsaufträge gibt es für Ärzte und Ärztinnen im Krankenhaus für Schwerstkranke und Sterbende? Und welche Behandlungsarrangements zeigen sich bei nicht heilbaren Patient*innen?

- Auf welches Wissen über eine palliativmedizinische Behandlung und palliative Versorgungsstrukturen im Krankenhaus und über den stationären Bereich hinaus greifen Ärzte und Ärztinnen zurück?

Mit der Untersuchung verfolge ich drei Ziele:

- Exploration ärztlicher Erfahrungen im Krankenhaus, um Forschungsergebnisse, welche die unzureichende Versorgung von Sterbenden in deutschen Krankenhäusern belegen [50], um Begründungen und dichte Beschreibungen [330] zu erweitern,

- Kontextualisierung der ärztlichen Erfahrungen durch eine Analyse der strukturellen und personellen Ressourcen für die Behandlungssituation im Krankenhaus,

- Zusammenführung der Analyseergebnisse im Sinne einer Theorie-Praxis-Brücke zur Versorgung Schwerstkranker und Sterbender im Krankenhaus. 
